\title{
Quand le cinéma grec passe à table
}

Les pâtes à l'écran

Meals in Greek Cinema: Pasta on Screen

Stéphane Sawas

\section{CpenEdition}

Journals

Édition électronique

URL : https://journals.openedition.org/ceb/6424

DOI : $10.4000 /$ ceb.6424

ISSN : 2261-4184

Éditeur

INALCO

Édition imprimée

ISBN : 978-2-85831-230-6

ISSN : 0290-7402

Référence électronique

Stéphane Sawas, «Quand le cinéma grec passe à table », Cahiers balkaniques [En ligne], Hors-série 2016, mis en ligne le 14 mars 2017, consulté le 06 juillet 2021. URL : http://journals.openedition.org/ ceb/6424; DOI : https://doi.org/10.4000/ceb.6424

Ce document a été généré automatiquement le 6 juillet 2021.

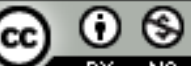

Cahiers balkaniques est mis à disposition selon les termes de la Licence Creative Commons Attribution - Pas d'Utilisation Commerciale 4.0 International. 


\title{
Quand le cinéma grec passe à table
}

\author{
Les pâtes à l'écran \\ Meals in Greek Cinema: Pasta on Screen
}

\author{
Stéphane Sawas
}

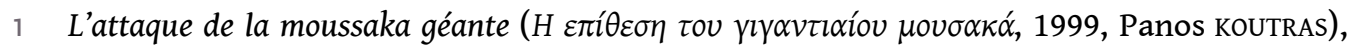

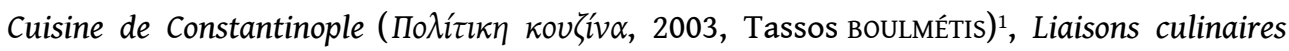

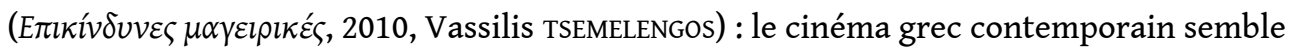
placé sous le signe de l'art culinaire. Ce constat initial invite à inscrire dans une profondeur diachronique la représentation cinématographique de l'acte de manger en Grèce, qui n'a guère retenu l'attention des chercheurs. En interrogeant cette représentation dans le cinéma grec de l'entre-deux-guerres et du $\mathrm{xxI}^{\mathrm{e}}$ siècle, un intéressant corpus se fait jour autour d'un plat particulier, certes essentiel dans l'alimentation des Grecs, mais pas immédiatement associé à la cuisine grecque : il s'agit des pâtes. Plat nourrissant et en apparence simple, qui ne nécessite pas la maîtrise d'un art culinaire sophistiqué, il est en outre protéiforme en fonction de l'assaisonnement prodigué, qu'il soit dégusté au restaurant ou dans le cercle familial. Ainsi, de 1924 à 2014, ce plat, sans jamais - ou presque - être le sujet principal des films du corpus révèle, au détour de séquences plus ou moins connues du public, les représentations contrastées de la société grecque à l'œuvre dans le septième art.

\section{Du muet à la dictature : grotesque et critique sociale}

2 Le premier film grec de fiction aujourd'hui présent dans les fonds d'archives cinématographiques (ROUVAS \& STATHAKOPOULOS, 2005, I, 24-25), les Aventures de Vilar ( $\mathrm{Ol}$

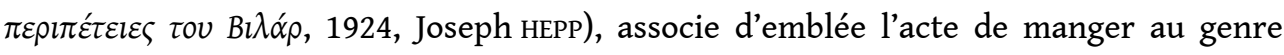
comique. La séquence finale de ce film présente un étrange banquet en plein air donné en l'honneur du mariage de deux nains, dont le caractère grotesque est peu présent dans le cinéma grec jusqu'à nos jours. Parmi les nombreux plats servis, les pâtes constituent l'apothéose : les convives se servent à même le plat et mangent les pâtes 
avec leurs mains sans utiliser de couverts, remplaçant parfois même leur assiette par leur chapeau.

3 Ce registre reste cependant marginal au sein de la production grecque. L'âge d'or de l'après-guerre, lié aux années troubles de la reconstruction, propose des représentations moins radicales, quoique souvent comiques, de l'acte de manger. Les pâtes apparaissent au centre d'un discours sur l'accès à l'alimentation des milieux favorisés et défavorisés au sein de l'ascension sociale promue par le cinéma dominant, associée au travail et au mariage (SAWAS, 2008a), dans des scènes qui ne manquent ni de tendresse ni de second degré. Prenons pour exemple une célèbre séquence de Moderne

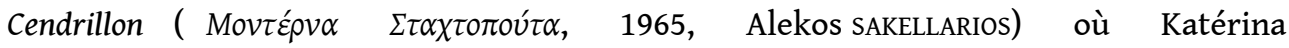
(Aliki vouYoukLAKI), qui vient de trouver du travail, téléphone à l'épicier de son quartier, sous le regard attendri de son nouveau patron Alexis Varnezis (Dimitris PAPAMichaïL). La commande qu'elle passe s'ouvre sur des pâtes qu'elle se propose de cuisiner à la bolognaise, avec de la viande hachée, signe d'une nouvelle aisance financière.

4 Chantre des milieux défavorisés, le cinéma populaire grec de l'après-guerre ne peut que difficilement tolérer un personnage qui dédaigne les nourritures auxquelles il a accès sans difficultés. Même si c'est un enfant, comme le montre le début du film Touche-à-

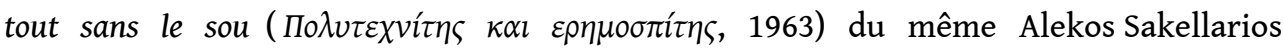
(adaptation d'une pièce de théâtre qu'il a écrite avec Christos yanNakopoulos): Thanassis Vengos y excelle en serveur qui subit les caprices du petit Yannakis (Vassilakis KAÏLAS). Cette séquence procède par répétitions multiples et le crescendo final, durant lequel les claquements de mains se multiplient, le rythme des questions s'accélère et le ton de la voix monte, renoue avec l'un des motifs favoris du cinéma comique depuis le muet, l'entartage - si ce n'est que la tarte à la crème est remplacée par une assiette de spaghetti.

5 Il n'est toutefois pour l'heure guère question de dégustation. Pourtant, les pâtes qui comptent parmi les plus célèbres du cinéma grec ne manquent pas de saveur : dans Une

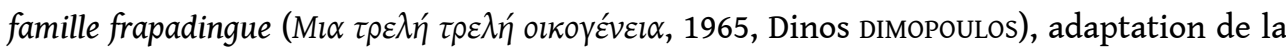

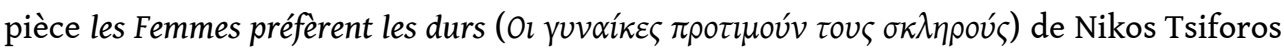
et Polyvios Vassiliadis (күRIAKOS, 2002, 256), l'une des comédies grecques les plus originales des années 1960 où l'humour confine à l'absurde, le jeune marié Andréas (Alekos ALEXANDRAKIS) rend folle de jalousie son épouse fantasque Mika (Jenny KAREZI) en commandant par téléphone à une mystérieuse Nana des pâtes aux anchois ( $\mu \alpha \kappa \alpha \rho o v \alpha ́ \delta \alpha$

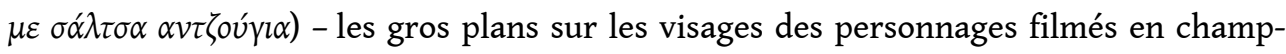
contrechamp soulignent du reste la stupéfaction générale. Signes de raffinement culinaire dans un milieu bourgeois, ces pâtes aux anchois sont explicitement mises en concurrence avec les pâtes italiennes (de retour de Venise, le protagoniste déclare s'être lassé des pâtes italiennes) et, dans la séquence suivante, sont dégustées à table avec la fourchette et la cuillère. La sauce aux anchois, sans être particulièrement grecque, évoque avec malice l'adultère (le faux adultère, toutefois, le personnage de Nana, interprétée par Lili Papayanni, étant la sœur du protagoniste) dans une comédie où la sexualité est l'objet de traitements métaphoriques, à la fois légers et ludiques, par exemple dans la séquence finale dansée.

6 Mais l'acte de manger n'est pas l'apanage des milieux favorisés et les pâtes se font parfois l'écho de tensions sociales aux implications politiques insoupçonnées, comme 


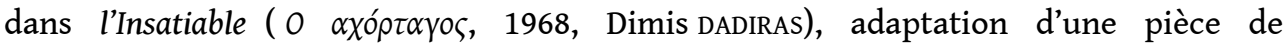
Dimitris Psathas (KYRIAKOS, 2002, 262) tournée au début de la dictature des colonels, ce qui n'est assurément pas sans conséquence. Dionyssis Papayannopoulos y campe un patron oisif et arrogant, comme le montre la scène restée célèbre où il commande un festin (avec pour commencer des pâtes) tout en prétendant ne vouloir que grignoter ( Je te l'ai dit, je vais grignoter aujourd'hui, je ne vais pas manger, je n'ai pas faim ${ }^{2}$, affirme-t-il pour clore le dialogue). La séquence suivante est plus éloquente, mais moins connue : ce n'est en effet pas le patron qui mange les pâtes, mais son futur employé interprété par Yannis Ghionakis (à même l'assiette sans faire usage de couverts ni de ses mains). Les allusions politiques sont à peine voilées : affamé, le personnage déclare ne pas être un humain, vendre son âme si on lui assure de quoi manger, et on parle de lui comme étant le premier à signer. Cet employé gravira par la suite l'échelle sociale, mais à la fin du film perdra la raison. Les allusions à la torture ${ }^{3}$ sont ici manifestes sous le prétexte de la farce, comme souvent dans le cinéma grec sous la dictature ; faut-il rappeler la généralisation de la déclaration de repentir dans la Grèce d'après-guerre? L'un des supplices dans les camps de torture ne consiste-t-il pas à donner à manger aux détenus en leur interdisant le recours aux couverts et parfois même aux mains? En décalage avec l'accompagnement musical qui évoque le burlesque muet, le contexte confère à cette séquence un aspect effrayant qui invite à revoir le cinéma comique de l'époque, trop souvent conçu de manière monolithique (SAWAS, 2008b, 219-221).

\section{De la transition démocratique au $\mathrm{XXI}^{\mathrm{e}}$ siècle : vers une diversification des genres}

7 L'après-dictature n'en est pas moins conflictuelle, mais cette fois de manière plus explicite à l'écran. Le terme « $\mu \alpha \kappa \alpha \rho o v \alpha ́ \delta \alpha$ » est en outre repris pour la première et

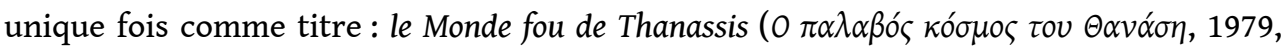
Dinos KATSOURIDIS) est un film à sketches dont le quatrième (placé au centre des sept) s'intitule les Pâtes ( $H \mu \alpha \kappa \alpha \rho o v \alpha ́ \delta \alpha)$. Les pâtes se retrouvent à plusieurs reprises dans les films dont Thanassis Vengos est l'interprète principal, qu'il fasse office de serveur dans

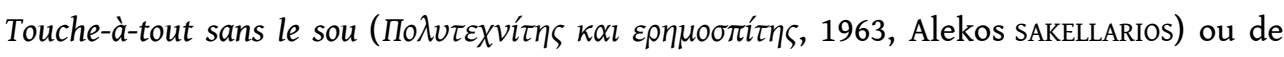

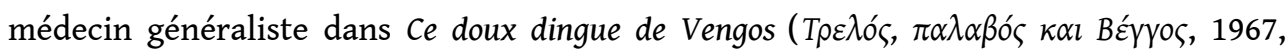
Thanassis VENGOS) où il retire sa fausse barbichette pour manger l'assiette de pâtes qui lui est offerte au domicile d'une patiente en s'exclamant : «Ah, pâtes immortelles ! " ${ }^{4}$. Il joue après la dictature dans une série de films caustiques où il critique la société de consommation et ses méfaits sur la Grèce qui connaît une fragile transition démocratique. Au début du sketch les Pâtes du film le Monde fou de Thanassis, il cherche du travail dans un contexte de déclin du cinéma, mais d'essor de la publicité. Inadapté aux méthodes et à l'esprit de cette dernière, le protagoniste ingère sur le tournage d'une réclame pas moins de sept assiettes de pâtes avec la fourchette et un morceau de pain, signe d'appartenance à un milieu populaire. Le sketch repose sur l'effet de mise en abyme associé à un efficace comique de répétition que rythment le signal du metteur en scène ( Play-back ») et le slogan ( Mangez des pâtes, mangez des pâtes ${ }^{5}$ ) rapidement interrompu par le même metteur en scène; un Vengos affamé, avatar de Karaghiozis, y donne à voir les failles d'une société en apparence lisse : les slogans invitent à manger les pâtes en famille (le deuxième couplet commence par «Les 
grands-parents en mangent, les petits-enfants aussi $\left.»^{6}\right)$, mais la satisfaction qui doit se montrer de manière ostentatoire et figée semble étrangère au protagoniste.

Les pâtes n'en restent pas moins l'un des plats préférés des enfants et des célibataires, comme le montrent deux des plus intéressants films grecs du début du xxI ${ }^{e}$ siècle : avec

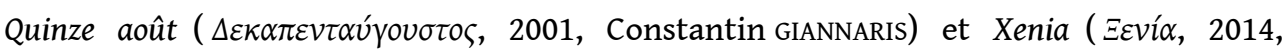
Panos KOUTRAS), les pâtes quittent la comédie pour apparaître dans des longs métrages qui relèvent d'autres genres cinématographiques.

Dans le film choral ${ }^{7}$ Quinze août, un squatteur s'introduit dans les appartements d'un immeuble d'Athènes dont les habitants sont partis, les uns en vacances les autres en pèlerinage. Par un vertigineux montage alterné, les destins entrent en résonance (HADJIPÉTROU, 2006). L'instabilité du cadre dans la séquence qui a posteriori relate la descente de l'escalier et l'entrée dans l'appartement, filmée caméra à l'épaule, conduit progressivement au personnage qui, attablé dans la cuisine, mange des pâtes sans sauce avec une fourchette seule (22'45-23'36), le jeune homme à peine sorti de l'adolescence (il manipule une voiture miniature à la fin de la séquence) aspirant à se trouver un nouveau foyer. Il est du reste intéressant de noter que l'interprète, Kostas Kotsianidis, n'est pas un acteur professionnel ; Grec d'URSS, il a tourné pour la première fois dans

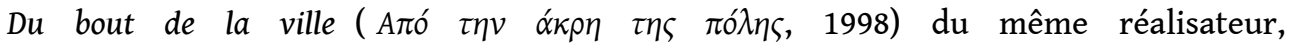
Constantin Giannaris (SAWAS, 2011, 11, 14). À la lueur de cette remarque, ce nouveau foyer change d'échelle pour s'étendre au moins à la Grèce dans son ensemble.

C'est également le cas dans Xenia: de la Crète à Thessalonique, les protagonistes de ce road movie, lui aussi interprété par de jeunes acteurs non professionnels, d'ascendance albanaise cette fois, sont deux frères au sortir de l'adolescence, Dany, seize ans, et Ody (Odysséas), dix-huit ans, apatrides de mère albanaise qui cherchent à être reconnus par leur géniteur pour obtenir la nationalité grecque. La présence des pâtes au début du film s'accorde bien aux thématiques traitées dans ce récit initiatique : nostalgie de l'enfance, quêtes identitaires et sociales de jeunes hommes célibataires. Mais les pâtes de ce film sont peut-être les plus étonnantes du corpus. Filmées en gros plan au début $\mathrm{du}$ film (10'47-10'53), trois assiettes de spaghetti au ketchup sont copieusement agrémentées de sucre en poudre par le frère cadet, ce qui ne suscite qu'une question sans réponse du colocataire dans la salle de séjour et un regard attendri de l'aîné sur la terrasse. Les acteurs expliquent que leurs parents albanais ayant l'habitude de préparer un dessert composé de pâtes et de sucre, ce plat surprenant pour beaucoup de spectateurs relève pour eux davantage d'une réminiscence culinaire que du mauvais goût $^{8}$. La Grèce, nouveau pays d'immigration, se fait une nouvelle fois le creuset de pratiques alimentaires et culturelles métissées.

\section{Conclusion}

11 Traversant les genres et les décennies, les pâtes, pourtant guère représentatives de la cuisine grecque, permettent de manière tantôt implicite tantôt explicite selon le contexte politique d'affirmer à l'écran des particularismes identitaires et de révéler des clivages politiques et idéologiques en mettant au jour par l'image animée les fonctionnements et les dysfonctionnements d'une société grecque en constante mutation. 


\section{BIBLIOGRAPHIE}

CHLEPOS-PANAJOTOPOULOS Anastasia-Katherine, 2008, la Cuisine de Constantinople : mémoire du passé et reflet d'une communauté, représentations littéraires et cinématographiques, mémoire de Master II dirigé par Stéphane Sawas, Paris : INALCO.

HADJIPÉTROU Hugo, 2006, Figures de mort et de résurrection dans Quinze août de Constandinos Giannaris, mémoire de maîtrise dirigé par Stéphane Sawas, Paris : INALCO.

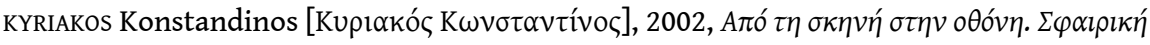

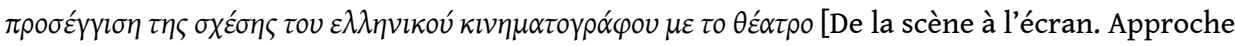
globale de la relation du cinéma grec au théâtre], Athènes : Aigokeros.

LABRECQUE Maxime, 2011, « Le film choral : l'art des destins entrecroisés », Séquences, $n^{\circ} 275$, p. 31-35.

NeVeu Pascal, 2013, " Makronissos (la Pierre) 》, in l'Amertume et la Pierre. Poètes au camp de Makronissos 1947-1951, Paris : Ypsilon, p. 269-309.

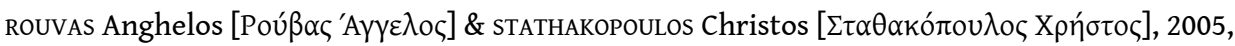

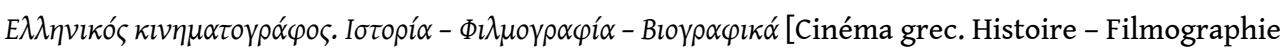
- Notices biographiques], Athènes : Ellinika Grammata.

SAWAS Stéphane, 2008a, « Le cinéma grec classique (1949-1967), un cinéma de l'évasion et de l'oubli », Revue des études néo-helléniques, nouvelle série, nº 4, p. 107-130.

SAWAS Stéphane, 2008b, «Les écrans grecs sous la dictature des colonels (1967-1974) : la grande rupture ", in MULLER Raphaël \& WIEDER Thomas (dir.), Cinéma et régimes autoritaires au XX ${ }^{e}$ siècle. Écrans sous influence, Paris : PUF/Éditions Rue d'Ulm, p. 213-227, 237-239, 261-264.

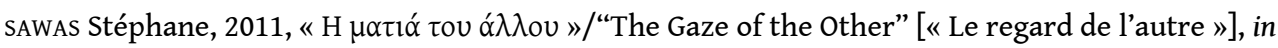

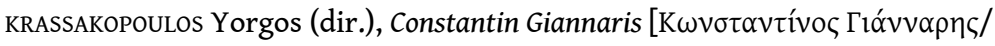
Constantine Giannaris], Thessalonique-Athènes : Thessaloniki International Film Festival Publications, p. 11-15.

\section{NOTES}

1. Ce film a été projeté hors de Grèce sous des titres aussi différents que A Touch of Spice ou Un ciel épicé. Voir Anastasia-Katherine chlEPOS-PANAJOTOPOULOS, 2008.

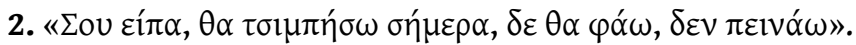

3. Sur la torture en Grèce, voir Pascal NEVEU, 2013.

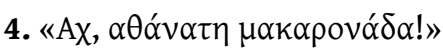

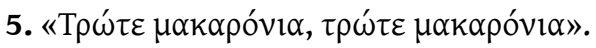

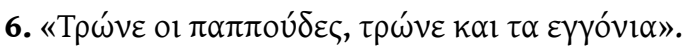

7. Sur le film choral, voir Maxime LABRECQUE, 2011.

8. Voir l'entretien avec les acteurs réalisé le 19 mai 2014 à Cannes dans le bonus intitulé «Rencontre avec Kostas Nikouli et Nikos Gelia» (7'46-8'28) du DVD du film commercialisé en France par Pyramide Vidéo en 2014. 


\section{RÉSUMÉS}

Sans être un plat caractéristique de la cuisine grecque, les pâtes révèlent à l'écran les principales tensions qui traversent la société grecque. Des Aventures de Vilar (1924) à Xenia (2014), les personnages, en mangeant des pâtes, expriment, en lien avec le contexte politique, leur désir de changer de vie.

Pasta is not a typical Greek dish, but its presence on screen reveals the main tensions of Greek society. From The Adventures of Vilar (1924) to Xenia (2014) the characters eating pasta express, in relation with the political context, their desire for another life.

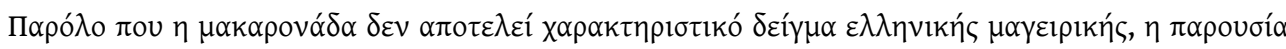

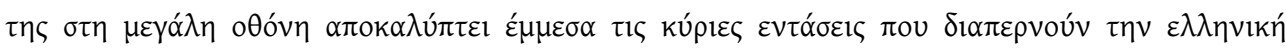

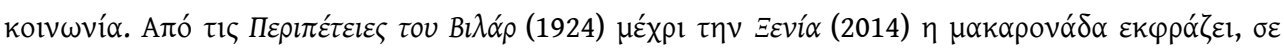

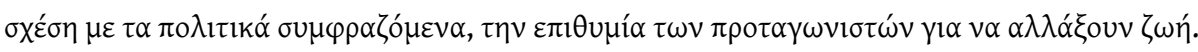

\section{INDEX}

Index géographique : Grèce

Thèmes : Cinéma, Société

motsclestr Yiyecek, Makarna, Temsil, Yunanistan, Yirminci yüzyıl, Yirmi birinci yüzyıl, Sinema, Toplum

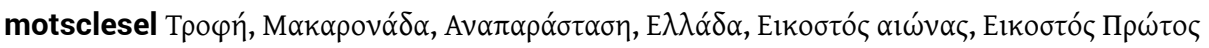

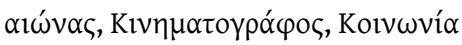

motsclesmk ХРАНА, ТЕСТЕНИНИ, ЗАСТАПЕНОСТ, ГРЦИЈА, ДВАЕСЕТТИОТ ВЕК, ДВАЕСЕТ И ПРВИОТ ВЕК, КИНО, ОПШТЕСТВО

Keywords : Food, Pasta, Representation, Greece, Twentieth Century, Twenty First Century, Cinema, Society

Mots-clés : nourriture, nourriture, pâtes, pâtes, représentation, représentation

Index chronologique : vingtième-et-unième siècle

\section{AUTEUR}

\section{STÉPHANE SAWAS}

Cerlom, Inalco, Sorbonne Paris Cité 